\section{Contact-controlled amoeboid motility induces dynamic cell trapping in 3D-microstructured surfaces $\dagger$}

\author{
Delphine Arcizet, $\$ \oint$ Sofia Capito, $\$$ Mari Gorelashvili, Carolin Leonhardt, Marion Vollmer, Simon Youssef, \\ Susanne Rappl and Doris Heinrich*
}

Received 7th April 2011, Accepted 7th November 2011
DOI: 10.1039/c1sm05615h

On flat substrates, several cell types exhibit amoeboid migration, which is characterized by restless stochastic successions of pseudopod protrusions. The orientation and frequency of new membrane protrusions characterize efficient search modes, which can respond to external chemical stimuli as observed during chemotaxis in amoebae. To quantify the influence of mechanical stimuli induced by surface topography on the migration modes of the amoeboid model organism Dictyostelium discoideum, we apply high resolution motion analysis in microfabricated pillar arrays of defined density and geometry. Cell motion is analyzed by a two-state motility-model, distinguishing directed cellular runs from phases of isotropic migration that are characterized by randomly oriented cellular protrusions. Cells lacking myosin II or cells deprived of microtubules show significantly different behavior concerning migration velocities and migrational angle distribution, without pronounced attraction to pillars. We conclude that microtubules enhance cellular ability to react with external 3D structures. Our experiments on wild-type cells show that the switching from randomly formed pseudopods to a stabilized leading pseudopod is triggered by contact with surface structures. These alternating processes guide cells according to the available surface in their $3 \mathrm{D}$ environment, which we observed dynamically and in steady-state situations. As a consequence, cells perform "home-runs" in low-density pillar arrays, crawling from pillar to pillar, with a characteristic dwell time of $\sim 75 \mathrm{~s}$. At the boundary between a flat surface and a 3D structured substrate, cells preferentially localize in contact with micropillars, due to the additionally available surface in the microstructured arrays. Such responses of cell motility to microstructures might open new possibilities for cell sorting in surface structured arrays.

\section{Introduction}

Amoeboid migration is studied preferentially in the social amoeba Dictyostelium discoideum or in neutrophils. This migration type is characterized by formation of stochastically generated pseudopods and proves to be extremely efficient in hunting for food or invadors. ${ }^{1}$ The fact that external stimuli affect the cellular search behavior is a major feature of this seemingly random cell motion. Membrane proteins sense and integrate external cues and transport signals to downstream effectors for specific cellular response or adaption. ${ }^{2,3}$

Center for Nanoscience (CeNS) and Faculty of Physics, Ludwig-Maximilians-Universität München, Geschwister-Scholl-Platz 1, 80539 München, Germany. E-mail: doris.heinrich@lmu.de

$\dagger$ Electronic supplementary information (ESI) available. See DOI: $10.1039 / \mathrm{c} 1 \mathrm{sm} 05615 \mathrm{~h}$

\$ These two authors contributed equally to this work.

$\S$ Current address: Institut de Biologie Structurale Jean-Pierre Ebel, Commissariat à l'Energie Atomique, 41 rue Jules Horowitz, 38027 Grenoble, France.
A prominent example for adaption to external stimuli is chemotaxis, where cells exposed to a gradient of chemoattractant molecules move up-gradient. ${ }^{4}$ During the past decade, many biochemical signalling pathways involved in chemotaxis have been elucidated for the motile cell archetype Dictyostelium discoideum. $^{5-8}$ The recruitment of key cytosolic proteins to the plasma membrane of the leading pseudopod triggers directional actin polymerization and subsequent migration towards the chemoattractant source. Analysis of cellular migration patterns under external stimuli reveals biased random search strategies with increased migration efficiency in terms of speed and directional persistence towards the chemotactic source. ${ }^{9}$

However, in the absence of chemotactic signaling, D. discoideum cells show a "basic motile behavior" ${ }^{10}$ The dynamics of this random migration mode is not fully understood yet. Even less advanced is the general understanding of amoeboid migration in the presence of three dimensional surface features.

Cell velocity and shape analysis led to simple models of amoeboid migration, describing it as a random walk. ${ }^{10}$ Recently, a refined view of amoeboid locomotion emerged, 
based on detailed analyses revealing correlations between cellular orientation and migration direction ${ }^{11-14}$ as well as motional persistence. ${ }^{15}$ The current understanding of basic amoeboid locomotion assumes two alternating motility modes: a random probing mode ( $\mathrm{rm}$-mode) and a fast, directed running mode (dir-run) ${ }^{16}$ Both modes originate from the spatiotemporal behavior of biochemical networks in the cell cytoskeleton: spontaneous actin polymerization near the plasma membrane results in random protrusions of multiple pseudopods (polypodial state, rm-mode), until one of them is reinforced, suppressing other pseudopods and temporarily stabilizing a directed run (monopodial state or dir-run). ${ }^{17}$ This two-statemotility in spontaneous amoeboid migration is strikingly similar to motion patterns found in large animals, ${ }^{18}$ which exhibit a local search mode and a long-distance running mode, and to the "run and tumble" motion of prokaryotic organisms, ${ }^{19}$ which emphasizes the universal character of this very efficient search strategy.

Moreover, D. discoideum cells were found to be capable of sensing physical constraints, an obviously vital asset for migration in a natural soil microenvironment. ${ }^{20}$ In fact, upon mutation of adhesion proteins, ${ }^{21}$ a complex topographical environment causes a drastic decrease in migration efficiency, in ways that go totally unnoticed on flat substrates. ${ }^{22}$ Studies on the effect of topographical cues on cytoskeleton dynamics have been facilitated by recent advances in microfabrication. The strong influence of prepatterned surfaces shaped as grooves or pillars on cell polarization and migration has been demonstrated for a variety of cell types. ${ }^{23-28}$ Mahmud et al. successfully sorted metastatic cells from healthy cells by directed migration in microstructured ratchets. ${ }^{29}$ Using antibody-coated microstructures in a fluidic system, rare circulating tumour cells could even be isolated from blood. ${ }^{30}$ Thus topographical microstructured setups have not only been demonstrated to constitute useful tools for medical assays, but they also allow the analysis of amoeboid migration in an environment simulating the in vivo situation. The differences between cell migration on flat substrates and within a three-dimensional topography are still unknown and remain a topic of ongoing investigations, for individual cells as well as for cell sheets. ${ }^{31}$

In this work, we analyse the migrational behavior of $D$. discoideum on three-dimensional and flat substrates in order to unravel the role of surface structures on motility modes. We find that microstructured surfaces are not sensed as obstacles, preventing cells from progressing efficiently, but rather lead to cell deflection or trapping, depending on the cells' initial motility mode and the number of surface structures in contact with the cells.

\section{Materials and methods}

We studied the migration modes of single $D$. discoideum cells in the vegetative state, in the absence of chemoattractant molecules and at low cell densities. Substrates for the migration assays were fabricated from the transparent polymer polydimethylsiloxane (PDMS) and consisted of micropillar arrays in combination with flat surfaces (which were used as a reference to study spontaneous cell motility in a homogeneous 2D environment).

\section{Cell culture and observation}

We used a mutant $D$. discoideum cell line expressing free GFP, imaged the cells by brightfield and fluorescence microscopy, and recorded time-lapse movies of 200 to 600 frames at a frame rate of $0.1 \mathrm{~Hz}$.

Dictyostelium discoideum culture and preparation for experiments. The axenic $D$. discoideum strain with GFP insertion, strain HG1694, ${ }^{32}$ and the myosin II-null mutant with LimE $\Delta$ cc-GFP, strain HS2205, ${ }^{33}$ were obtained from Dr Günther Gerisch (MPI for Biochemistry, Martinsried, Germany). The cells were grown at $21{ }^{\circ} \mathrm{C}$ in $\mathrm{AX} 2$ medium, supplemented with the antibiotic gentamicin at a concentration of $20 \mu \mathrm{g} \mathrm{m}^{-1}$ (G-418, Biochrom AG, Berlin, Germany). Myosin II-null mutants were additionally supplemented with the antibiotic blasticidin at a concentration of $20 \mu \mathrm{g} \mathrm{ml}^{-1}$. During cell culture in Petri dishes, the cell concentration was kept below $40 \%$.

A benomyl $\left(\mathrm{C}_{14} \mathrm{H}_{18} \mathrm{~N}_{4} \mathrm{O}_{3}\right.$, Sigma-Aldrich, Germany) solution in phosphate buffered saline (PBS) was used as the microtubule depolymerization agent. The solution was added to the $D$. discoideum WT cell suspension $30 \mathrm{~min}$ before measurements at a concentration of $100 \mu \mathrm{M}$.

For microscopy experiments, cells were harvested from the Petri dishes by three successive washing steps with $17 \mathrm{mM} \mathrm{K}-\mathrm{Na}$ phosphate buffered saline, adjusted to $\mathrm{pH}=6.0$ (PBS, Sigma Aldrich, Steinheim, Germany) and supplemented with $18 \mathrm{~g} \mathrm{l}^{-1}$ maltose (Maltose monohydrate, Karl Roth GmbH, Karlsruhe, Germany). The cells suspended in the maltose-PBS solution were then added progressively to the Ibidi observation chamber (uncoated 8-well, Ibidi, Martinsried, Germany) and let to settle down, until a concentration of 5 to 10 cells per 125 by $125 \mu \mathrm{m}$ (camera field of view) was achieved. This concentration ensures enough cells for statistics and few enough cells to exclude any cell-cell signaling, which would bias spontaneous migration.

Microscopy. The experimental chamber was mounted onto a Zeiss Axiovert 200M microscope and kept at $T=21{ }^{\circ} \mathrm{C}$. Both brightfield and fluorescence images were acquired every $10 \mathrm{~s}$ with an exposure time of $150 \mathrm{~ms}$ by an Andor iXon Dv885 CCD camera (Andor, Belfast, UK), triggered by the corresponding AndorIQ software.

\section{PDMS microstructures}

All substrates used for migration assays were fabricated from transparent polydimethylsiloxane (PDMS) after casting, crosslinking and unpeeling from a silicon wafer-based master obtained by photolithography (Fig. 1G-I). These PDMS arrays of 10-12 $\mu \mathrm{m}$ high pillars of $4 \mu \mathrm{m}$ diameter are arranged in a regular lattice or in a network of varying pillar density. Outside the pillar fields, large areas of the same chemical composition (PDMS) are used as a reference flat substrate.

Pillar structures and flat surfaces exhibit the same chemical composition (PDMS) throughout this study. In addition to that, both the wall and the base of the pillars, as well as the flat area between two pillars, exhibit homogeneous surface properties. Cells are not subject to any chemical attraction. Due to their low adhesivity, $D$. discoideum cell motility should not be strongly 

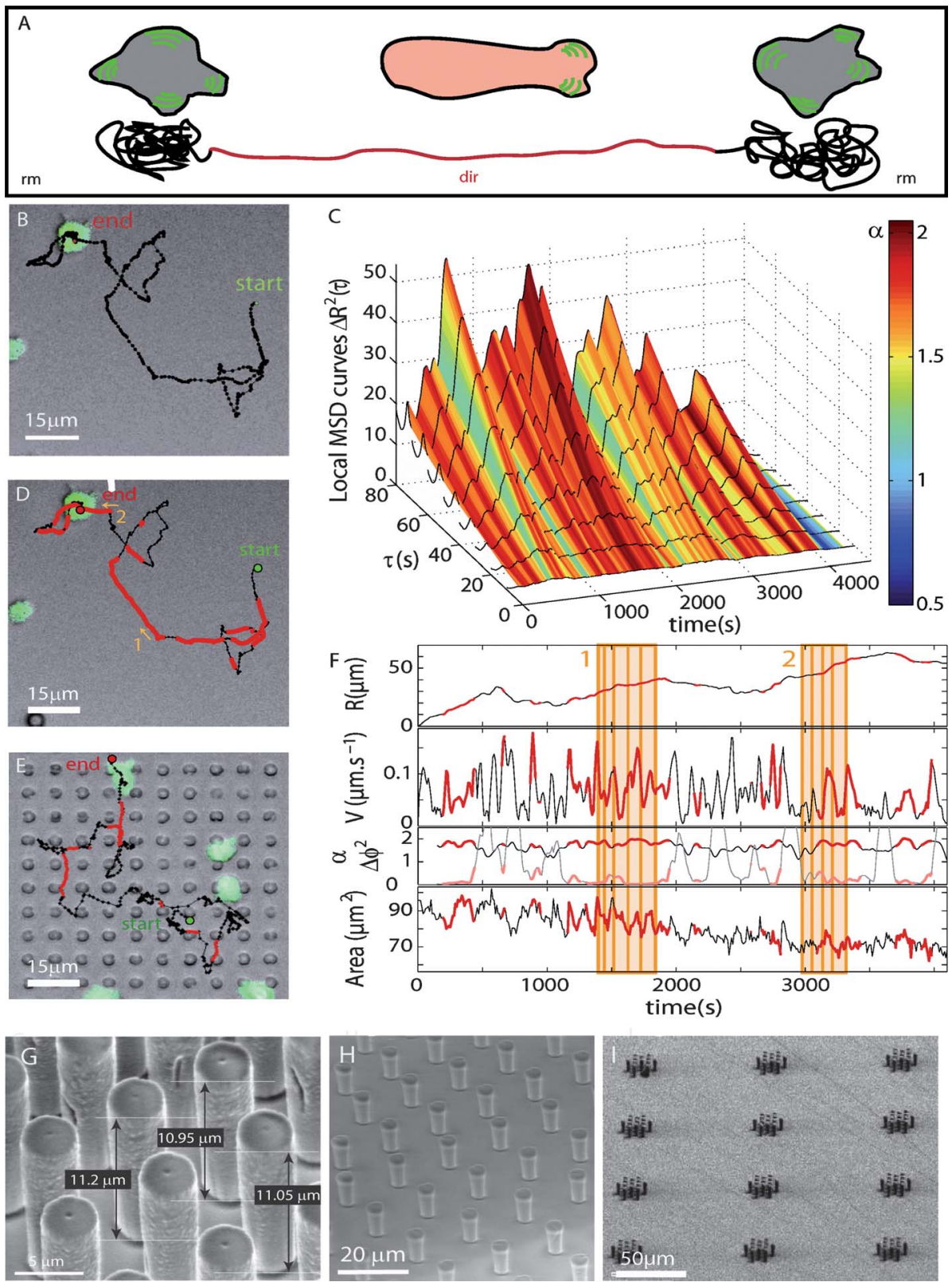

Fig. 1 Trajectory splitting into the two motion modes by the TRAnSpORT algorithm and PDMS microstructures. (A) Scheme of the transition from the random migration mode, the $r m$-mode (grey), where the cell exhibits multiple pseudopods, to a dir-run (red) characterized by one leading pseudopod (actin protrusions, represented in green). (B) Cell center-of-mass track on a flat surface before analysis. (C) Landscape of local MSD functions, calculated for each time $t_{i}$ of the experiment (color-coded for $\alpha$, the value of the power-law fitting exponent). The high $\alpha$ values, together with a high angular persistence, set the time points for which the motion is directed (dir-runs). (D and E) Cell track on flat PDMS (D) and within a rectangular micropillar array (E) after splitting into the 2 motion modes: dir-runs (red) and $r m$-modes (black). (F) Time course of the different variables defining the cell motion: cell position $R(t)$, instantaneous speed $V(t)$, local MSD exponent $\alpha(t)$ (black) together with the standard deviation of the angle variations $\Delta \phi^{2}(t)$ (grey) and area. The red portions of the curves indicate dir-runs. The windows highlighted in orange correspond to dir-runs (numbered 1 and 2 on image D). The speed oscillations are closely related to the area oscillations, emphasized by the orange lines. (G) SEM view (close up) of $4 \mu \mathrm{m}$ diameter micropillars, with measured heights given after correction for the e-beam angle of $45 \mathrm{deg}$. (H-I) SEM micrographs of $4 \mu \mathrm{m}$ diameter pillars, arranged in a regular square network $(\mathrm{H})$, and in low-density arrays shaped as pillar islands (I).

influenced by surface chemistry, but the comparison between purely $2 \mathrm{D}$ and quasi $3 \mathrm{D}$ substrates on the same sample - consisting of the same material - ensures that any difference observed in cell motility modes can only be attributed to the well-defined micron-scale topography and not to different chemical properties of the surface.
Master fabrication. To obtain a master for PDMS molding, we used standard clean room microlithography procedures, and relied on the protocols presented by Steinberg et al. ${ }^{26} \mathrm{~A} 3$-inches silicon wafer (Si-Mat, Landsberg/Lech, Germany) was cleaned under nitrogen flow, covered with approximately $5 \mathrm{ml}$ of a SU810 negative photoresist (Microchem, distributed by MicroResist 
Technology, Berlin, Germany), and spin-coated at $3000 \mathrm{rpm}$ for $30 \mathrm{~s}$ after progressive acceleration. A progressive soft bake (1 min at $65^{\circ} \mathrm{C}$ and $2 \mathrm{~min}$ at $85-90^{\circ} \mathrm{C}$, with slow cooling down to room temperature) was followed by UV-illumination in a mask aligner (Süss MicroTec, Garching, Germany) through a Chromium mask (ML\&C, Jena, Germany). This mask bears the structures transferred to the resist. Illumination times varied between 3 and $5 \mathrm{~s}$ according to the size of the desired structures. After a progressive hard bake $\left(1 \mathrm{~min}\right.$ at $65^{\circ} \mathrm{C}$ and $2 \mathrm{~min}$ at $85-90{ }^{\circ} \mathrm{C}$, with slow cooling down to room temperature), the unlit, and hence non-crosslinked photoresist, was washed away by two successive development baths in a SU8-specific developer (MicroResist Technology, Berlin, Germany). The photoresist structures obtained were stored at room temperature and exhibit stable properties for several months.

Preparation of the PDMS structures. To ensure proper PDMS unmolding, the resist master was silanized: vapour deposition of a fluorosilane $(1 \mathrm{H}, 1 \mathrm{H}, 2 \mathrm{H}, 2 \mathrm{H}$-perfluorooctyltrichlorosilane, ABCR, Germany) was achieved under vacuum in one hour.

The PDMS base was mixed vigorously with the cross-linking agent at a 1 to 10 ratio (Sylgard 185 Silicon Elastomer Kit, Dow Corning, MI, USA). After degassing for $30 \mathrm{~min}$ under vacuum, 2-3 $\mathrm{ml}$ of PDMS were poured on the Si-photoresist master, and degassed again for 15 to 30 minutes under vacuum. PDMS crosslinking was obtained after 3 to 5 hours at $65^{\circ} \mathrm{C}$.

After cutting and peeling the PDMS structures off the master, the samples were exposed to Argon plasma for $30 \mathrm{~s}$, to make the surface hydrophilic (Plasmaanlage "Femto", Diener Electronic, Nagold, Deutschland). They were then immediately transferred to the observation chambers (uncoated 8-wellplates, Ibidi, Germany), immersed in PBS solution, and kept sterile until further use with cells.

\section{Cell tracking and trajectory analysis}

All cell positions, areas and perimeters were retrieved from the acquired fluorescence images by a homemade plugin for the Image J analysis software (W. S. Rasband, U. S. National Institutes of Health, Bethesda, USA, http://imagej.nih.gov/ij/). The pillar positions were obtained from brightfield images with the same plugin. The cell trajectories were then processed by a homemade Matlab algorithm (The Mathworks Inc. Natick, USA), which is able to retrieve the local motion modes characterizing cell migration (Fig. 1C and F). Furthermore, the information retrieved on cell contact with pillars enables the study of the influence of micron-scale obstacles on $D$. discoideum motility modes.

Migration track retrieval by the single cell tracker plugin. Our single fluorescent cell tracking plugin has been adapted to follow the rapid motion of $D$. discoideum amoebae. This algorithm detects fluorescent cells as clusters of more than $n$ bright pixels above an intensity threshold $I_{0}$ and in closer proximity than a distance $\varepsilon$. All three parameters $\left(n, I_{0}, \varepsilon\right)$ can be freely adjusted to yield the most reliable cell clustering results. Tracking of each brightness cluster throughout the movie is achieved by finding, from one frame to the next, the most proximate cluster (in space and intensity), which is then identified as the same object. Each brightness cluster represents a single $D$. discoideum cell, and yields its center of mass position $(X, Y)$, area $(A)$ and perimeter $(P)$ as a function of experiment time.

From brightfield images, the pillar positions can be determined employing the same plugin: $\uparrow$ after image treatment (noise reduction) and thresholding, a precise map of the pillar outlines on each time frame is retrieved. This map is then used for determining and analyzing the cell-to-pillar contact (see "Contact evaluation"). Also, pillar positions were used to estimate a potential stage motion (long-term drift or short-term small oscillations) and to subtract it from the overall cell motion before analysis.

Cell motion analysis by the TRAnSpORT algorithm. Our TRAnSPORT (Time-Resolved Analysis for the Splitting Of Random Trajectories) routine ${ }^{34-36}$ performs cell motion analysis, since it is capable of distinguishing two motility modes, one of them exhibiting high directional persistence and the other mode being an isotropic, diffusive-like random walk.

For each time point $t_{i}$, representing the center of a rolling window of $M=30$ points, a local mean square displacement $\Delta R_{i}^{2}$ (1-MSD, Fig. 1C) is calculated as a function of the time lag $\tau_{k}=$ $k \delta t$ as follows:

$$
\begin{aligned}
\Delta R^{2}\left(t_{i}, \tau_{k}\right) & =\Delta R_{i}^{2}(k) \\
& =\frac{1}{M-k+1} \sum_{j=-M / 2}^{(M / 2)-k}\left(\boldsymbol{R}\left(t_{i+j}+k \delta t\right)-\boldsymbol{R}\left(t_{i+j}\right)\right)^{2}
\end{aligned}
$$

where $\delta t$ is the frame rate and $\boldsymbol{R}\left(t_{i}\right)=\left(X\left(t_{i}\right), Y\left(t_{i}\right)\right)$ are coordinates of the center of mass. $T=M \delta t$ is the duration of the rolling window. We already showed that the resulting algorithm time resolution is of the order of $T / 4 .{ }^{34}$ The l-MSD functions are then fitted by power laws $f_{i}(\tau)=A_{i} \tau^{\alpha_{\mathrm{i}}}$, with the exponents $\alpha_{i}$ bearing information about the motility state at each point $i$ (Fig. 1C and $\mathrm{F}$ ).

The angle persistence function $\Delta \phi_{i}$ is calculated from the values of the velocity angle $\phi_{i}=\left(v_{x, i}, v_{y, i}\right)$ :

$$
\begin{aligned}
\Delta \phi\left(t_{i}, \tau_{k}\right) & =\Delta \phi_{i}(k) \\
& =\sqrt{\frac{1}{M-k+1} \sum_{j=-M / 2}^{(M / 2)-k}\left(\phi\left(t_{i+j}+k \delta t\right)-\phi\left(t_{i+j}\right)\right)^{2}}
\end{aligned}
$$

The 1-MSD exponent and angle persistence values allow for a dissection of the trajectory into two different motility states: if $\alpha_{i}$ is close to 2 and $\Delta \phi_{i}$ is close to zero, the motion is classified as directed (or ballistic), and the cell is in a dir-run (see Fig. 1D-F, red phases). Otherwise, the motion is called random probing (or non-directed), and the cell is in an rm-mode. This is summarized by a binary probability for directed motion $p_{\text {dir }}$

$$
p_{\text {dir }}=\left\{\begin{array}{l}
1 \text { if }\left[2-\sigma_{\alpha} \leq \alpha \leq 2\right] \wedge\left[0 \leq \Delta \phi \leq \sigma_{\phi}\right] \\
0 \text { otherwise }
\end{array}\right.
$$

The directed parts of the trajectory correspond to phases during which the amoeba crawls at a quasi-constant speed in a quasi-preserved direction. An angular deviation of $\sigma_{\phi}=3 \sigma_{\alpha}$ is

T Pillars appear as very bright disks, due to a light guiding effect along their axis. 
allowed, so that dir-runs do not depend on slow changes in the motion direction (the value of $\sigma_{\alpha}$ is set to 0.3 , to ensure correct discrimination between the dir- and $r m$-modes). Using both criteria, the exponent and angle persistence values are necessary due to the uncertainty of the $\alpha$-value arising from the limited number of points in the rolling window.

Contact evaluation. The aim is to reliably measure the number of pillars that the cell is in contact with for each image. This is called the total contact index $I_{\text {cont }}$ of a single cell during its trajectory. We extract dwell times, which are the periods for which the cell has been in contact with one or more pillars.

The probability for a cell $c$ to be in contact with a pillar $p$ in its vicinity depends on the distance between the cell and pillar, and more specifically, on the fraction of the cell area which is close to this pillar. More formally, we introduce a pairwise contact index $i_{\text {cont,c-p }}$ which reflects this probability. In order to calculate this index, we define two parameters: $\varepsilon$ (the critical cell distance or proximity in units of pixels) and $N_{\min }$ (the minimum cell area in units of pixels that has to be closer than $\varepsilon$ to the pillar). If $n_{\text {cell }}$, the number of cell pixels in proximity to the pillar, is greater than $N_{\text {min }}$, then the index $i_{\text {cont,c-p }}$ is set to 1 . Otherwise, if $n_{\text {cell }}$ is smaller than $N_{\min }$, the index value is set to $n_{\text {cell }} / N_{\min }$.

The total contact index for a given cell $c$ in a given frame $j$ is the sum over all pairwise indices corresponding to different pillars:

$$
I_{\text {con } t}^{c}(j)=\sum_{p} i_{\text {cont }, c-p}
$$

This yields the total number of pillars the cell is in contact with. Running this analysis frame by frame allows for correlating the cell-to-pillar contact with the cell motility.

Through the use of the contact index, the statistics on the motion characteristics (instantaneous speed, $\alpha$ exponent and dirrun lifetimes) can be split into specific categories, corresponding to the phases during which the cell is in contact with none, one or two (or more) pillars. Such a distinction is crucial to understand the influence of topographical cues on cell migration.

\section{Results and discussion}

\section{Cell migration analysis by a 2-state motility model}

In this work, we investigate the influence of $3 \mathrm{D}$ environments on cellular migration modes. Using standard photolithography procedures and polymer molding, we fabricated arrays of pillars of $4 \mu \mathrm{m}$ diameter and 10-12 $\mu \mathrm{m}$ height, made of transparent polydimethylsiloxane (Fig. 1G-I). Pillar structures and flat surfaces exhibit exactly the same chemical composition, ensuring that any observed effect is of topographical origin only. In order to compare the migration of $D$. discoideum cells on flat surfaces and in microstructured pillar fields, the recorded migration trajectories of fluorescent cells were analyzed with high spatial and temporal resolution by a two-state motility model.

First, image processing is used to capture the cell motion of GFP-labeled D. discoideum cells. The cell center of mass is identified and tracked throughout the movie. We then analyze individual cell migration trajectories by our local-MSD-algorithm: a predefined time window slides across the migration track and allows for the calculation of a local mean square displacement (MSD) ${ }^{34}$ (see also Materials and methods). The migration mode is assigned as directed (dir-run) when two conditions are fulfilled: (i) the local MSD function is close to a quadratic law with the exponent $\alpha$ of the power-law fit close to 2 , and (ii) the angular persistence of the trajectory is high, with a standard deviation $\Delta \varphi$ of the velocity angle close to 0 . If these conditions are not fulfilled, the cell is in a diffusive-like random probing ( $\left.\mathrm{rm}^{-}\right)$mode (Fig. 1A-F). Each track is dissected into random probing states and directed runs.

\section{Cell migration on a flat surface}

The global MSD functions calculated over the entire trajectories yield information about the cell migration type as a whole. Typical example functions are given in Fig. 2N-Q (corresponding tracks shown in the inset). The motion is superdiffusive at short time scales and diffusive at long time scales with a typical cross-over time from one regime to the other. But this global analysis can only give a rough representation of cell migration as a random walk which, at short time scales, involves periods of rather directed migration. While the double fitting of the global MSD functions can only yield one characteristic cross-over time $\tau_{\mathrm{C}}$, our high resolution analysis of local motion types enables separation of the contributions of different migration modes, and analysis of the distribution of their precise characteristics: local MSD exponent, velocity and lifetime.

Spontaneous migration on a flat substrate is characterized by alternating phases of directed and random motion modes (Fig. 1A). Two directed runs (shown in red in Fig. 1D and E) are separated by a random phase during which the cell slows down, probes its environment and repolarizes. A new dir-run in another direction starts after this isotropic reorientational phase. After trajectory splitting by our local MSD analysis (Fig. 1C and F), one can retrieve the phase durations, together with specific migration parameters such as velocities of the dir-runs and diffusion coefficients for the $r m$-modes (Fig. 1F). The advantage of our time-resolved analysis lies within the fact that the parameters are only evaluated during the corresponding motion phases.

During migration on a 2D surface without external stimulus (see Fig. $1 \mathrm{~B}$ and D, and Fig. 2B, F, J and N), WT cells show $35 \%$ of dirruns, which are randomly distributed without preferred direction in the $X-Y$ plane (Fig. 1D). Their lifetime decays over $\left\langle\tau_{\mathrm{d}}\right\rangle=140 \mathrm{~s}$ and the runs cover a distance of $11-21 \mu \mathrm{m}$, which corresponds to 1-2 cell lengths. The speed distribution function exhibits a Gaussian shape, characterized by a well-defined mean speed of $\left\langle V_{\text {dir }}\right\rangle=0.076 \mu \mathrm{m} \mathrm{s}^{-1}$ (averaged over all measured cells (Fig. 2B)). Each dir-run is composed of 2 to 4 cycles of expansion/retraction of the cell body, which also results in speed oscillations (Fig. 1F). By contrast, the $r m$-mode speed is significantly smaller $\left(\left\langle V_{\mathrm{rm}}\right\rangle=\right.$ $\left.0.057 \mu \mathrm{m} \mathrm{s}^{-1}\right)$ and its distribution is best fitted by a log-normal function, indicating that numerous processes of various origins and time scales are involved here. Furthermore, rm-modes are typically concentrated within regions of less than $7 \mu \mathrm{m}$ in diameter.

\section{Cell migration modes in regular micropillar arrays}

We applied the same analysis to cell migration of three different types of $D$. discoideum within a dense rectangular micropillar 


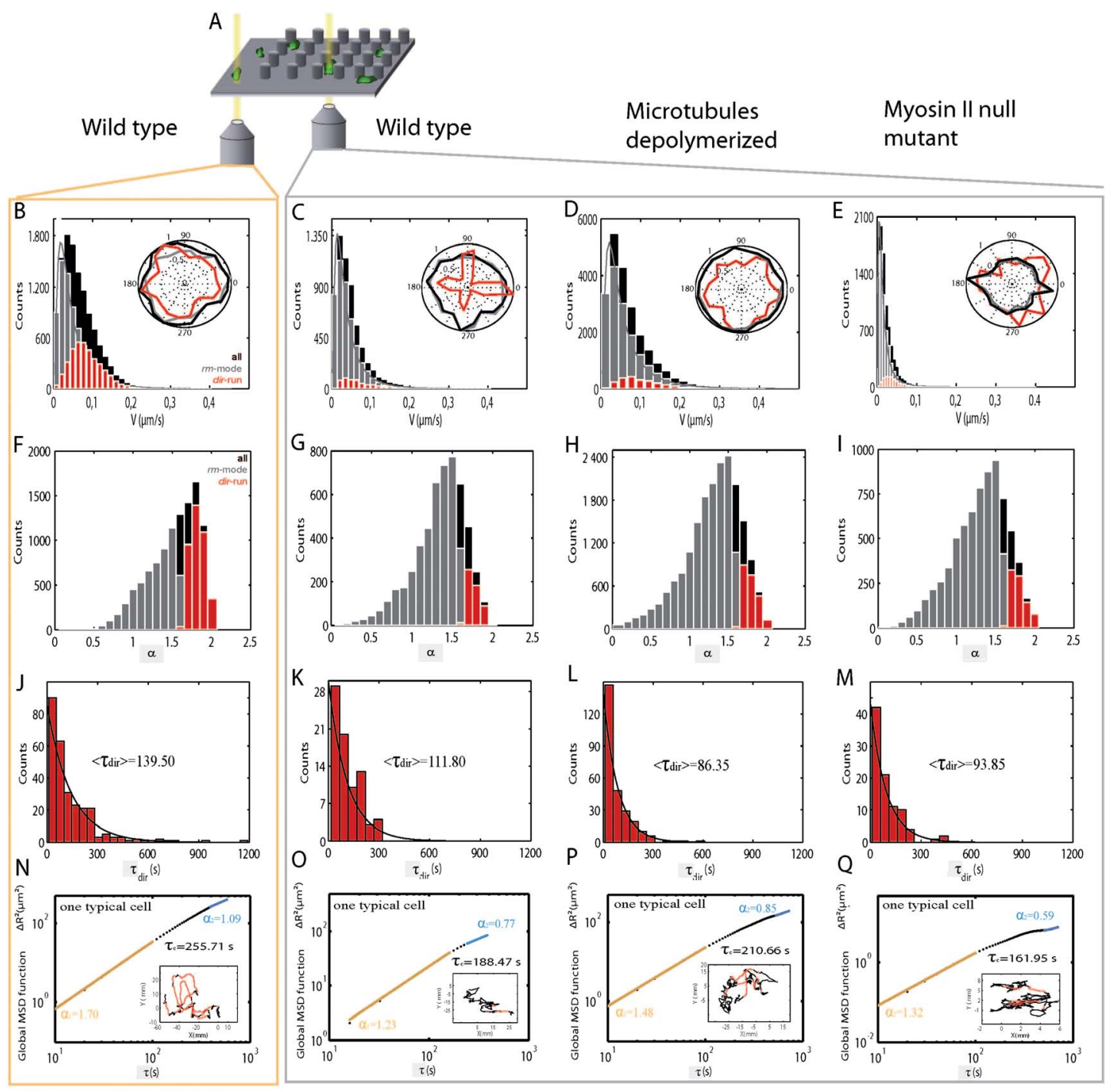

Fig. 2 Spontaneous amoeboid motility on a flat substrate $v s$. topography-guided migration within a square lattice of micropillars. (A) Scheme of the experimental setup: comparison of $D$. discoideum migration on flat and microstructured areas, both identical in chemical composition. (B-E) Instantaneous speed distributions, resulting from all analyzed cells on flat PDMS $(N=27$ cells) and within this micropillar array wild type $(N=13$ cells), benomyl treated cells $(N=27$ cells) and myosin II-null mutants $(N=14$ cells) (black: all data points, red: dir-runs, grey: $r m$-modes). Velocities during $r m$ modes are fitted by a log-normal distribution. Angle distributions of the velocity vector are shown in the insets (normalized to one). (F-I) Distributions of the exponent $\alpha$, power-law fit of the local MSD functions, revealing the amount of dir-runs and $r m$-modes in the overall cell motion. (J-M) Distributions of the dir-run lifetimes, with fits by a simple exponential. (N-Q) Global MSD function, calculated on a single cell track shown in the inset: the short time/long time power law trends in the global MSD are highlighted in orange and blue respectively. The track has been split into dir-runs (red) and $r m$-modes (black).

network of $4 \mu \mathrm{m}$ pillar-to-pillar distance, which is about one third of a typical cell diameter (Fig. 1E). Our measurements reveal a strong decrease in dir-run frequency compared to flat surfaces (Fig. 2, and Table 1): dir-runs represent only 10-12\% of the cell motion (vs. $32 \%$ on flat). This is also reflected in a smaller average $\alpha$ value of the local MSD analysis.

In WT cells, we find a smaller average velocity $\left\langle V_{\mathrm{rm}}\right\rangle$ of $0.043 \mu \mathrm{m} \mathrm{s}^{-1}$ in pillar structures as compared to $0.057 \mu \mathrm{m} \mathrm{s}^{-1}$ on flat substrates. However, the velocities during directed runs $\left\langle V_{\text {dir }}\right\rangle$ are larger $\left(0.082 \mu \mathrm{m} \mathrm{s}^{-1}\right.$ as compared to $0.076 \mu \mathrm{m} \mathrm{s}^{-1}$ on flat substrates). The most striking effect appears in the velocity angle distribution: while the $r m$-modes remain randomly oriented, the $d i r$-runs reflect the network geometry (Fig. 2). So the presence of dense and regularly spaced micropillars heavily modifies $D$. discoideum migration modes: the decrease in frequency and duration of the dir-runs results in a much more confined cell motion. The rare directed runs are strongly guided along the lattice axes of the micropillar network, resulting in a biased run from pillar to pillar along these preferred directions (see ESI S2 $\uparrow$ ).

Benomyl-treated cells, lacking microtubules, show in general larger velocities. Compared to WT cells in pillar fields, they do not stay in dir-runs as long as WT cells and exhibit rather random orientation. We find that the angle distribution of the dir-runs does not reflect the pillar geometry any longer.

Myosin-II null mutants show significantly reduced velocities in general. They are rather confined by neighbouring pillars due to 
Table 1 Parameters describing the cell motility on a flat substrate $v$ s. a square network of dense pillars (4 $\mu \mathrm{m}$ pillar-to-pillar distance), corresponding to Fig. 2

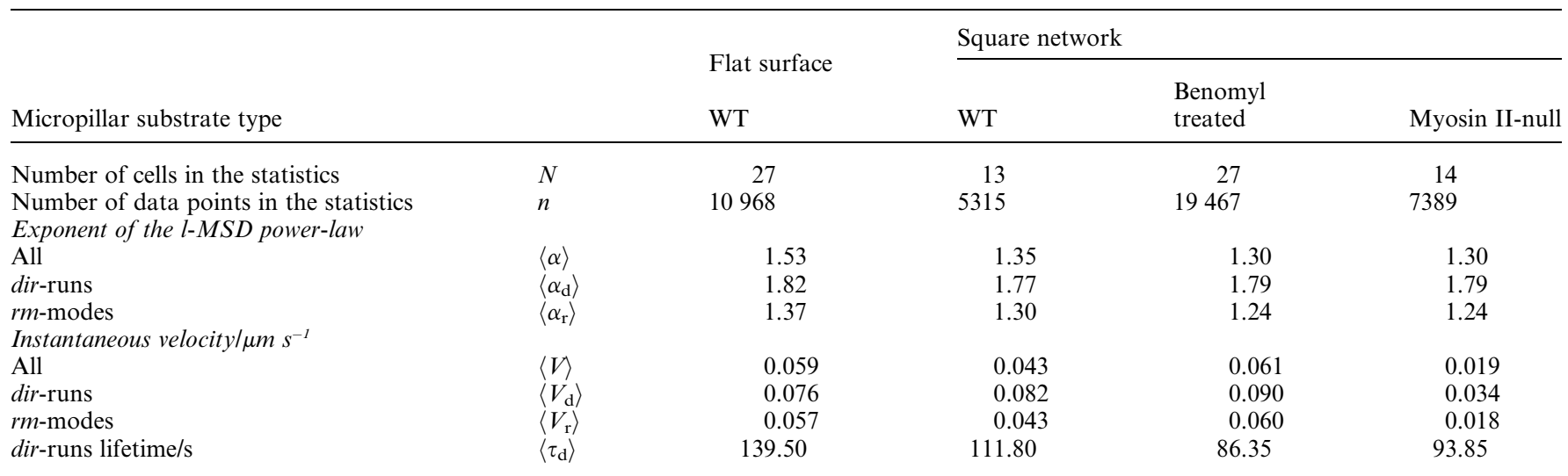

their extended cell size, as they cannot retract their tale effectively, and therefore do not show large displacements. Here, we find slight reflection of the pillar network geometry in the random migration angle distribution.

\section{Migration modes at the flat/structured interface}

In order to identify if significant trends in cell migration directed towards flat or structured surfaces can be revealed, we investigated motility modes and preferred cell localization at the interface between flat substrates and pillar fields. For that purpose, we designed low-density micropillar islands of about $50-100 \mu \mathrm{m}$ in diameter, each consisting of 13 pillars. The islands are separated by wide flat areas (Fig. 1I). A contact index, i.e. the number of pillars a cell is in contact with along its trajectory (see Materials and methods), is defined. We find contact indices ranging from 0 to 4 , depending on the inter-pillar distances. For cells migrating within a pillar island with several pillars in their vicinity, one subpopulation of cells moves from pillar to pillar with dwell times between 50 and $200 \mathrm{~s}$, exhibiting mostly dir-runs (Fig. S3C $\uparrow$ and $3 \mathrm{E}$ and F) whereas another cell population stays in contact with the same pillar for up to 500-900 s, mostly in the rm-mode (Fig. S3A $\dagger$ ).

For inter-pillar distances larger than the typical cell diameter, where a single cell cannot touch two pillars at the same time, a striking "stick-and-go" type of motion is observed: the cells "jump" from pillar to pillar, exhibiting long dir-runs $\left(\left\langle l_{\mathrm{d}}\right\rangle=\right.$ $8.2 \mu \mathrm{m}$ ) thereby covering long distances (Fig. S1 and Movies S6 and $\mathrm{S} 7 \dagger$ ). This manifests the transition regime between 2D random migration and a migration that is modified by surface structures. In contrast, when the inter-pillar distance is slightly smaller than one cell diameter, cell motility is reduced by temporary trapping. Cells in contact with two pillars do not show any net displacement and cell locomotion can be stalled for durations of $900 \mathrm{~s}$ and longer (Fig. S2 and Movie S8†).

To quantify statistical dwell times, we averaged over the entire cell population (Fig. 3A). The dwell time distribution can be fitted by a double exponential $1-\beta \exp \left(-\tau / \tau_{1}\right)-(1-\beta) \exp (-\tau /$ $\left.\tau_{2}\right)$, which reflects the existence of two characteristic dwell times (fit calculated for the cumulative distribution). The main dwell time $\tau_{1}$, representing around $77 \%$ of the touching events, is found to be $\sim 74 \mathrm{~s}$, and the second characteristic dwell time is $\tau_{2} \approx 290 \mathrm{~s}$ (corresponding to $23 \%$ of the touching events). This confirms that the vast majority of cells do not stay in contact for much more than one minute: most of the touching events are transient, along the "stick-and-go" motion of the cell. This proves that, in the case of low-density obstacles, cells are not stalled when they contact surface structures. Although pillars can be considered to be cell attractors, they should not be assigned as static traps. The dynamic trapping effect probably arises from feedback loops induced by internal signalling. When the cell is close to a pillar, pseudopods which are randomly protruded in all three dimensions during the $r m$-phase are more likely to touch the surface of $3 \mathrm{D}$ pillars than flat areas. Since it has been proven that a protrusion which touches a surface becomes a leading pseudopod with higher probability, ${ }^{37}$ the likelihood for a cell to exit the random probing mode and to subsequently enter a new directed run, induced by a stabilized pseudopod, is increased in the vicinity of a micropillar (Fig. S3C and E $\dagger$ ). However, the dwell time distribution includes values of up to $900 \mathrm{~s}$, which reflects the rare but still observable very long touching events that were mentioned previously.

\section{Cell ensemble partitioning between structured and unstructured surfaces}

So far, we discussed migration trajectories of individual cells in the presence of pillars. We now take a different view and consider the steady-state situation of an ensemble of cells partitioning between pillar fields and unstructured areas. We assume that after a sufficient amount of time (typically 1-3 hours), a detailed balance of amoebae migrating in and out of the micropillar fields is reached. As shown in Fig. 3C, we perform a time and ensemble average for 27 cells in pillar structures for 1-3 hours and find that $22 \%$ of the cells are in contact with pillars. This percentage of cells in contact is now compared to the percentage of substrate in the vicinity of pillars (i.e. the area where cells can be in contact with a pillar) with respect to the total surface area. As indicated in Fig. 3B and C, we define the contact area by a circular region around a pillar of $r=7 \mu \mathrm{m}$ in width, i.e. half the typical cell diameter of a $D$. discoideum cell. This area accounts for $8 \%$ of the total 2D surface: the "relocalization" factor is thus $0.22 / 0.08=$ 2.75 ( $c f$. ESI $\dagger$ ). Accordingly, almost three times as many cells are in contact with pillars than would be expected for equal 

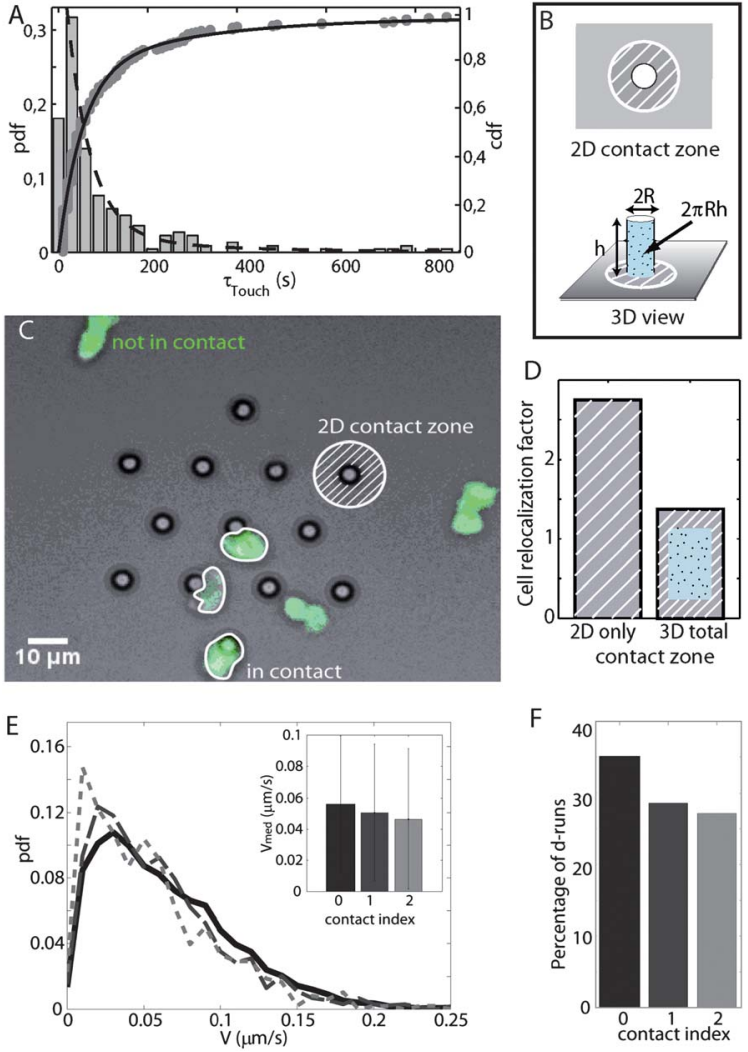

Fig. 3 Cell partition between the flat substrate and islands of micropillars. (A) Dwell times: probability distribution function (pdf, grey bars) and cumulative distribution function (cdf, grey dots) of dwell-times spent by cells in contact with pillars, for the whole population of cells migrating within and in-between islands of micropillars. The cdf is well fitted by a double exponential $1-\beta \exp \left(-\tau / \tau_{1}\right)-(1-\beta) \exp \left(-\tau / \tau_{2}\right)$ (black line) with characteristic times $\tau_{1}=74 \mathrm{~s}$ and $\tau_{2}=289 \mathrm{~s}$, where $\beta=0.77$ indicates that $77 \%$ of the touching events happen for a typical time $\tau_{1}$. (B) Scheme of the 2D vs. 3D surface available around each pillar: (top) 2D projection of a pillar (radius $R=2 \mu \mathrm{m}$ ), with the hatched circle representing the zone within contacting distance for cells (radius $r=7 \mu \mathrm{m}$ ); (bottom) 3D view of the pillar with its walls offering an additional surface of $2 \pi R h$ (dotted blue). (C) Typical image underlining the cell relocalization effect: the zones within pillar-contacting distance (flat areas circumscribed around the pillars within which a cell can touch at least one pillar) represent only $25 \%$ of the 2D surface and yet, 3 out of 6 cells $(50 \%)$ are in contact with pillars (white cell borders), the remaining 3 cells not being in contact with pillars (no cell borders indicated). This partition can only be explained by the $3 \mathrm{D}$ surface offered by the pillar walls, which yields an equivalent surface ratio of $50 \%$ in this specific case. (D) Cell relocalization factor, i.e. the percentage of cells in contact with pillars divided by the percentage of surface area within contact distance to pillars, averaged over all 27 measured cells. Without counting the surface available in the third dimension, this factor is almost 3 . Taking the $2 \mathrm{D}$ contact zone and $3 \mathrm{D}$ surface offered by pillar walls into account, the total available surface in the vicinity of pillars increases drastically, which brings the relocalization factor close to 1: the cell distribution over the substrate exactly reflects the total available surface. (E) Velocity distribution and median velocity: compared to the reference on the flat surface (black, plain), the velocity distribution shows a slight shift towards low velocity values when the cells touch one (dashed, grey) or two pillars (dotted, light grey), which can also be seen in the median velocity values, shown in the inset (black: flat surface, grey: contact with 1 pillar, light grey: with 2 pillars). (F) Percentage of dir-runs: the frequency of dir-runs also decreases slightly distribution according to the available 2D surface. However, if we additionally consider the vertical surfaces of the pillar walls (surface area: $2 \pi R h$ ), this situation changes drastically: in this case, the ratio of contact area $\left(2 \pi R h+\pi(R+r)^{2}\right)$ to total area is $16 \%$, which, considering experimental errors, corresponds to the percentage of cells in contact with pillars: the "relocalization" factor becomes $0.22 / 0.16 \approx 1$ (Fig. 3D). Hence we find that, on average, all cells are distributed equally with respect to the effective surface area within the three-dimensional microstructures. Given the length scales involved, it is natural to notice that cells do not distinguish between horizontal and vertical surfaces during adhesion and migration. We show that $2 \mathrm{D}$ projections, which are our familiar way of seeing things, can be misleading and even hide fundamental 3D-induced effects.

\section{Conclusion}

In summary, we investigated amoeboid migration on microstructured surfaces as a prototype example of migration in threedimensional, natural environments. We found that the twodimensional trajectories of Dictyostelium discoideum cells encountering micropillars show distinct differences from the basic two-state motility model on flat substrates.

\section{Amoeboid migration modes are altered by the presence of 3D structures}

According to the initial motility state, we discriminate two types of cell behavior upon pillar contact: randomly moving cells stay in contact with pillars, whereas fast moving cells in a directed run phase get deflected by the pillars. Cells migrating inside pillar arrays exhibit a characteristic stick-and-go behavior, if the pillar distance is large compared to the cell diameter. Furthermore, the partitioning of cells between pillar fields and flat areas allows us to conclude that cells distribute equally according to the total available surface in three dimensions.

\section{Amoeboid cells migrate by maximizing contact with available surfaces}

All our observations are consistent within the framework of a two-state migration model, where cells switch between phases of random pseudopod formation and phases where a single pseudopod is stabilized. Our experiments suggest that the switching from a randomly formed pseudopod into a stabilized pseudopod is enhanced by surface contact. This additional feature, in which the protrusion is reinforced independently of the spatial orientation of the contacted surface, guides cells through 3D environments.

In cells lacking microtubules or myosin II, we find significantly different behavior. Cells lacking microtubules do not show a pronounced attraction to pillars, although we find larger velocities during dir-runs. We conclude that microtubules enhance cellular ability to react with external 3D structures. In cells lacking myosin II, we find strongly decreased migration

with contact index. But on average, the cells keep moving directionally (more than $25 \%$ of the migration modes) and fast (more than $75 \%$ of the migration velocity on $2 \mathrm{D}$ ), even when contacting $3 \mathrm{D}$ microstructures. 
velocities in both migration modes and a slight guiding behavior in the random mode due to the neighboring pillars.

All in all, the best frame to analyze our results on wild-type cells seems to be the model of contact-reinforced motility, or, more exactly, pseudopod stabilization upon surface contact with a subsequently increased probability to become the leading pseudopod. This leading pseudopod then sets the direction for a new dir-run, which, in the case of pillar contact, will be directed towards the pillar.

The effect of pillars, however, should always be considered as dynamic, as the overall cell motion remains a quasi-random walk. The emission of random protrusions is probably not biased by the presence of microstructures, while the dir-runs are. In the resulting mechanism of contact guidance, it is a stable cell polarization (the presence of a stable actin front at the leading edge due to surface-generated feedback loops and intracellular signalling) that leads to motional persistence towards microstructures. Amoeboid migration is hence guided by surface contact and is "haptotactic" in this sense. Considering the microstructure of soil, which is the natural habitat of Dictyostelium discoideum cells, contact-controlled motility is an advantageous strategy for amoebae to reach surface structures during their search for food, since bacteria preferably grow in dips and niches.

\section{Possible advances for quantitative medical assays}

As demonstrated here, experiments using micro-structured surfaces in combination with quantitative analysis of cell motility provide a useful tool to uncover details of cell-surface interactions and their coupling to cell motility.

In a medical context, this concept offers several opportunities for invasion assays, allowing for cell sorting on a large scale. By adding chemical gradients, which can be done in a very sophisticated way by now, ${ }^{38}$ cells could be separated even more efficiently. The assay could easily be scaled up for insertion of whole cell sheets or even tissue parts to investigate collective cell motion phenomena. Related to this, the influence of edges and corners on cytoskeleton activity remains to be investigated in future experimental and theoretical work and should reveal crucial clues for the understanding of how topographical details of a substrate affect cell migration, as described for wall-like structures in ref. 39 .

\section{Acknowledgements}

We thank E. Sackmann (TU München, Germany) and B. Lindner (Humboldt University, Berlin) for fruitful discussions, J. Rädler (LMU München, Germany) for helpful comments on the manuscript, S. Schulz (University of Heidelberg, Germany) for his kind help on the microlithography protocols, P. Altpeter and P. Paulitschke (LMU, Germany) for the SEM pictures. D.H. acknowledges funding from the Deutsche Forschungsgemeinschaft (grant HE5958-2-1) and from the Volkswagen-Foundation (grant I85100). C.L. acknowledges financial support by the Elite Network of Bavaria (International Doctorate Program NanoBioTechnology).

\section{References}

1 P. Friedl, Curr. Opin. Cell Biol., 2004, 16, 14-23.

2 J. Dalous, E. Burghardt, A. Muller-Taubenberger, F. Bruckert and G. Gerisch, et al., Biophys. J., 2008, 94, 1063-1074.

3 C. M. Lo, H. B. Wang, M. Dembo and Y. L. Wang, Biophys. J., 2000, 79, 144-152.

4 P. N. Devreotes and S. H. Zigmond, Annu. Rev. Cell Biol., 1988, 4, 649-686.

5 C. A. Parent and P. N. Devreotes, Science, 1999, 284, 765-770.

6 N. L. Jeon, H. Baskaran, S. K. W. Dertinger, G. M. Whitesides and L. Van De Water, et al., Nat. Biotechnol., 2002, 20, 826-830.

7 C. L. Manahan, P. A. Iglesias, Y. Long and P. N. Devreotes, Annu. Rev. Cell Dev. Biol., 2004, 20, 223-253.

8 L. Song, S. M. Nadkarni, H. U. Bodeker, C. Beta and A. Bae, et al., Eur. J. Cell Biol., 2006, 85, 981-989.

9 R. J. Petrie, A. D. Doyle and K. M. Yamada, Nat. Rev. Mol. Cell Biol., 2009, 10, 538-549.

10 D. R. Soll, D. Wessels, P. J. Heid and H. Zhang, J. Muscle Res. Cell Motil., 2002, 23, 659-672.

11 J. C. Del Alamo, R. Meili, B. Alonso-Latorre, J. RodriguezRodriguez and A. Aliseda, et al., Proc. Natl. Acad. Sci. U. S. A., 2007, 104, 13343-13348.

12 D. Heinrich, S. Youssef, B. Schroth-Diez, U. Engel and D. Aydin, et al., Cell Adhes. Migrat., 2008, 2, 58-68.

13 Y. T. Maeda, J. Inose, M. Y. Matsuo, S. Iwaya and M. Sano, PLoS One, 2008, 3, e3734.

14 Y. Iwadate and S. Yumura, J. Cell Sci., 2008, 121, 1314-1324.

15 L. Li, S. F. Norrelykke and E. C. Cox, PLoS One, 2008, 3, e2093.

16 I. Weber, Eur. J. Cell Biol., 2006, 85, 915-924.

17 T. D. Pollard and G. G. Borisy, Cell, 2003, 112, 453-465.

18 S. Benhamou, Ecology, 2007, 88, 1962-1969.

19 H. C. Berg and D. A. Brown, Nature, 1972, 239, 500-504.

20 P. Friedl, S. Borgmann and E. B. Brocker, J. Leukocyte Biol., 2001, 70, 491-509.

21 S. Cornillon, L. Gebbie, M. Benghezal, P. Nair and S. Keller, et al., EMBO Rep., 2006, 7, 617-621.

22 E. Ponte, E. Bracco, J. Faix and S. Bozzaro, Proc. Natl. Acad. Sci. U. S. A., 1998, 95, 9360-9365.

23 J. Tan and W. M. Saltzman, Biomaterials, 2002, 23, 3215-3225.

24 M. T. Frey, I. Y. Tsai, T. P. Russell, S. K. Hanks and Y. L. Wang, Biophys. J., 2006, 90, 3774-3782.

25 J. P. Kaiser, A. Reinmann and A. Bruinink, Biomaterials, 2006, 27, $5230-5241$.

26 T. Steinberg, S. Schulz, J. P. Spatz, N. Grabe and E. Mussig, et al., Nano Lett., 2007, 7, 287-294.

27 M. Ghibaudo, L. Trichet, J. Le Digabel, A. Richert and P. Hersen, et al., Biophys. J., 2009, 97, 357-368.

28 D. Wessels, H. Vawter-Hugart, J. Murray and D. R. Soll, Cell Motil. Cytoskeleton, 1994, 27, 1-12.

29 G. Mahmud, C. J. Campbell, K. J. M. Bishop, Y. A. Komarova and O. Chaga, et al., Nat. Phys., 2009, 5, 606-612.

30 S. Nagrath, L. V. Sequist, S. Maheswaran, D. W. Bell and D. Irimia, et al., Nature, 2007, 450, 1235-1239.

31 X. Trepat, M. R. Wasserman, T. E. Angelini, E. Millet and D. A. Weitz, et al., Nat. Phys., 2009, 5, 426-430.

32 D. Gabriel, U. Hacker, J. Kohler, A. Muller-Taubenberger and J. M. Schwartz, et al., J. Cell Sci., 1999, 112, 3995-4005.

33 D. Manstein, M. Titus, A. DeLozanne and J. Spudich, EMBO J., 1989, 8, 923-932.

34 D. Arcizet, B. Meier, E. Sackmann, J. O. Rädler and D. Heinrich, Phys. Rev. Lett., 2008, 101, 248103.

35 J. Mahowald, D. Arcizet and D. Heinrich, ChemPhysChem, 2009, 10, $1559-1566$.

36 C. Pelzl, D. Arcizet, G. Piontek, J. Schlegel and D. Heinrich, ChemPhysChem, 2009, 10, 2884-2890.

37 D. Wessels, H. Vawter-Hugart, J. Murray and D. Soll, Cell Motil. Cytoskeleton, 1994, 27, 1-12.

38 B. Meier, A. Zielinski, C. Weber, D. Arcizet, S. Youssef, T. Franosch, J. O. Rädler and D. Heinrich, Proc. Natl. Acad. Sci. U. S. A., 2011, 108(28), 11417-11422.

39 E. Sackmann, F. Keber and D. Heinrich, Annu. Rev. Condens. Matter Phys., 2010, 1, 257-276. 